\title{
Quantification of Seasonal Variation in Daily Physical Activity in Individuals with Heart Failure and Implantable Cardioverter Defibrillator/Cardiac Resynchronisation Therapy Devices
}

Michael J Shoemaker, ${ }^{1}$ Amanda Ferrick, ${ }^{1}$ Cathryn Fischer, ${ }^{1}$ Colin Schuurman, ${ }^{1}$ Kelly Cartwright, ${ }^{2}$ Jessica McLeod ${ }^{2}$ Emily Schuman, ${ }^{2}$ Ashley Van Dam, ${ }^{2}$ Michael G Dickinson ${ }^{3}$

1. Department of Physical Therapy, Grand Valley State University, Grand Rapids, MI, USA; 2. Department of Physical Therapy, Spectrum Health, Grand Rapids, MI, USA; 3. Spectrum Health, Frederik Meijer Heart and Vascular Institute, Grand Rapids, MI, USA

$\mathrm{P}$ urpose: Understanding seasonal variation of daily physical activity (PA) in patients with heart failure (HF) has important implications for planning and interpretation of clinical trials, but the presence and magnitude of this seasonal variation in daily PA have yet to be established. The purpose of the present study was to determine the presence and magnitude of seasonal variation in daily PA in community-dwelling individuals with HF using several analytic approaches. Methods: Retrospective chart review of patients with HF and Medtronic implantable cardioverter defibrillator/cardiac resynchronisation therapy (ICD/CRT) devices. Data included in analyses (autocorrelation, analysis of covariance, one-way analysis of variance) were clinical characteristics, the patient activity measure of daily PA from the ICD/CRT devices, temperature and hours of daylight over the 1-year period of 1 November $2017-31$ October 2018. Results: One hundred and sixty-eight patients were included. Visual analysis and autocorrelation demonstrated seasonal variation in daily PA. Daily PA seasonal difference between winter and summer months was 0.4 hours per day/24 minutes per day/2.8 hours per week/14.9\%. This seasonal effect on daily PA is significantly greater in those with $\leq 8$ comorbid conditions and an overall activity level of $>2.2$ hours per day compared to those with multiple comorbidities and low overall activity ( 0.7 versus 0.1 hours per day, respectively). Conclusions: The present study affirms the seasonality of daily PA in a cohort of patients with HF and ICD/CRT devices and reveals a disproportionate seasonal effect on those with fewer comorbidities and higher overall activity levels. Seasonal variation should be accounted for when interpreting change in daily PA in clinical practice and when designing and interpreting results of clinical trials investigating interventions to improve daily PA.

\section{Keywords}

Heart failure, physical activity, seasonal variation

Disclosure: Michael J Shoemaker, Amanda Ferrick, Cathryn Fischer, Colin Schuurman, Kelly Cartwright, Jessica McLeod, Emily Schuman, Ashley Van Dam and Michael G Dickinson have nothing to disclose in relation to this article.

Review Process: Double-blind peer review. Compliance with Ethics: All procedures for this study were followed in accordance with the responsible conduct of research, institutional review board (IRB) committee approval, the Helsinki Declaration of 1975 and subsequent revisions, and a waiver of informed consent approved by the IRB due to the retrospective record review nature of the study.

Authorship: The named authors meet the International Committee of Medical Journal Editors (ICMJE) criteria for authorship of this manuscript, take responsibility for the integrity of the work as a whole, and have given final approval for the version to be published.

Received: 3 May 2019

Accepted: 4 November 2019

Citation: Heart International. 2019;13(2):31-7

Corresponding Author: Michael Shoemaker, 301 Michigan Street N.E., Suite 200, Grand Rapids, MI, 49503 USA. E: shoemami@gvsu.edu

Support: No funding was received for the publication of this article.
In individuals with heart failure (HF) who also have implanted cardioverter defibrillators and/or cardiac resynchronisation therapy (ICD/CRT) devices, low daily physical activity (PA) levels lead to increased risk of mortality, poor aerobic capacity and lower health-related quality of life. Improving daily PA in these individuals may, therefore, be an important clinical endpoint; however, designing interventional trials has proved to be problematic due to the confounding effect of seasonal variation. While there has been a significant amount of research devoted to the effect of seasonal weather changes on daily PA in individuals with chronic obstructive pulmonary disease (COPD), , 3-5 community-dwelling older people, ${ }^{6-11}$ healthy adults ${ }^{12-17}$ and functionally impaired older people, ${ }^{18}$ there is a paucity of similar research in individuals with HF. Therefore, a better understanding of the way in which season affects daily PA in individuals with HF is needed to assist clinical trial design and in clinical interpretation of changes in daily PA over time.

Previous research on seasonal variation in daily PA is limited in several important ways: 1) wide variation in the definition of daily $P A_{1}{ }^{7-9,18} 2$ ) heterogeneity of measures of daily $P A_{1}{ }^{1-11,14-20}$ 3) short daily PA sampling periods $5,7-10,12,16$ and 4) use of non-longitudinal designs that compared separate groups of subjects from different seasons using relatively short sampling periods. 1,2,1,1,1,15,17 Therefore, the purpose of the present study was to determine the presence and magnitude of seasonal variation in daily PA in community-dwelling individuals with HF through the use of several analytic approaches.

\section{Methods}

Study design and measure of daily physical activity

This retrospective chart review included patients with HF and a range of Medtronic ICD/CRT devices (Medtronic, Dublin, Ireland) living in West Michigan. The objective was to examine change in daily PA data compared with average temperature and hours of daylight over the 1-year period of 1 November 2017-31 October 2018. Medtronic ICD/CRT devices include a single-axis 
accelerometer that record daily PA in 1-minute increments for every minute a patient is moving at an equivalent of 70-80 steps per minute. This recorded activity is then graphically reported in hours per day as 'patient activity' on the Cardiac Compass ${ }^{\mathrm{TM}}$ (Medtronic, Dublin, Ireland) device interrogation report for the preceding 14 months. The patient activity measure demonstrates strong correlation and agreement with triaxial accelerometer measures of daily $\mathrm{PA}^{21}$ and therefore provides a unique opportunity for longitudinal daily PA analysis. The present study did not include individuals with devices from other manufacturers due to differences in measurement mechanisms and associated algorithms for calculating and reporting PA.

\section{Patient selection}

A list of potentially eligible patients managed by the Spectrum Health Cardiac Device Clinic was generated from the Optima database for patients with HF who were seen for an in-clinic device check between 1 November 2017 and 16 January 2018. This would allow for the possibility that patient activity data were available for the target sampling period due to the rolling 14-month period of data stored in the device.

Inclusion criteria were based on patients with the following characteristics: 1) a diagnosis of HF due to ischaemic or non-ischaemic cardiomyopathy, 2) New York Heart Association (NYHA) class I-IV, 3) management by the Spectrum Health Medical Group, and 4) Medtronic ICD/CRT device. Patient records were excluded if the patient: 1) did not live within a 50 mile radius of a weather reporting station; 2 ) did not have patient-activity data available during the selected 1-year sampling frame (1 November 2017-31 October 2018); 3) a documented limitation in ambulation such as wheelchair use, severe orthopaedic condition, stroke or other neurologic disease, or amputation; 4) a left ventricular assist device; 5) greater than five hospitalisations for any reason; or 6) a major cardiovascular or orthopaedic surgical procedure during the sampling frame such as coronary artery bypass grafting, aortic aneurysm repair, joint replacement, amputation, or spinal surgery.

\section{Data collection procedures}

The investigators reviewed electronic medical records of included patients for clinical characteristics (age, sex, aetiology of cardiomyopathy, NYHA class, device type, left ventricular ejection fraction [LVEF], number of comorbidities [measured as a simple count of chronic conditions], number and length of hospitalisations [based on local/in-system electronic health record data], nearest weather reporting station [based on home address]). Bi-monthly data points were recorded (start of month and middle of month) for the 1-year sampling period using visual estimation of the patient activity measure from an electronic copy of the Cardiac compass device interrogation report. The Cardiac Compass report provides graphic and numeric information on numerous parameters, including device status, thoracic impedance, defibrillations, episodes and durations of atrial fibrillation and ventricular fibrillation, heart rate variability, average nightly heart rate, and patient activity. Visual estimation of the patient activity measure was performed by adjusting the size (i.e., zooming in/out) of the electronic copy of the graph to match the y-axis (which represents hours of activity per day) to that of the ruler.22,23 At each data point, the recorded value was that which approximated the 2-week average around each point measured to the nearest tenth. ${ }^{22,23}$ Although inter-rater reliability and validity of this method has been previously established, ${ }^{22,23}$ it was re-established for the present study using 20 randomly selected records and three investigators to reflect the current investigators' inter-rater reliability and measurement validity for the present study. The three investigators independently visually estimated, with a ruler, the amount of activity during the final week prior to device interrogation to allow for comparison with the average patient activity that is calculated by, and reported in, the Cardiac Compass report only for the week preceding the interrogation.

Weather data for relevant weather reporting stations for 1 November 2017-31 October 2018 were obtained from the Local Climate Dataset (www.ncdc.noaa.gov/cdo-web/datasets), including the maximum, minimum and average temperature. Total daylight hours were obtained from the United States Naval Observatory (https://aa.usno.navy.mil/ data/docs/Dur_One Year.php). The study protocol was approved by the Spectrum Health Institutional Review Board.

\section{Statistical analysis}

Inter-rater reliability for the visually-estimated measurement of patient activity was analysed using the intraclass correlation coefficient. ${ }^{2,1}$ concurrent validity was analysed using the Pearson correlation coefficient to quantify the correlation between the visually estimated measurement of patient activity and the average patient activity calculated and reported for the final week on the Cardiac Compass device interrogation report.

Data analyses were comprised of multiple approaches to identify the presence and magnitude of seasonal variation in patients with $\mathrm{HF}$ and ICD/CRTs. These analytic approaches reflect the varied methods used in previous studies of seasonal variation in daily PA. Autocorrelation was used to identify the presence of seasonality in patient activity. By comparing the autocorrelation plots of average temperature, daylight and patient activity, inferences can be made about the presence of seasonality and whether the seasonality of patient activity corresponds to the seasonal trends for temperature and daylight hours. Autocorrelation is a technique that can be used for detecting seasonality in time-series data by measuring the correlation of time-series data with itself by calculating the correlation between any two points in time across a specified number of time lags. ${ }^{24}$ Random data will have values near zero across the plot. The time/number of lags between peaks observed on the plot indicate the duration of each season. ${ }^{24}$ Repeated measures ANCOVA modelling was used to determine the presence of seasonality (i.e., a cubic main effect of time) and to determine the influence of clinical characteristics on seasonal variation in patient activity. ${ }^{13}$ Relevant clinical characteristics were identified using a Pearson correlation coefficient matrix of clinical characteristics and maximum seasonal difference in patient activity. Ultimately, comorbidities and overall mean annual activity level were included in ANCOVA modelling. Comorbidities were dichotomised based on the mean number of comorbidities ( $\leq 8$ as determined by the mean for the overall sample and an apparent breakpoint in the scatterplot of comorbidities and seasonal difference in patient activity) and overall activity level according to a previously established threshold that differentiated daily PA patterns ( $\leq 2.2$ hours per day). ${ }^{22}$ The magnitude of seasonal differences in patient activity were analysed using dependent t-tests for the overall sample and a one-way ANOVA to test the difference in seasonal patient activity between groups of patients with different combinations of clinical characteristics used in the ANCOVA. Statistical analyses were completed using IBM Statistical Package for the Social Sciences V.24 (SPSS, Chicago, IL, USA).

\section{Results}

A total of 378 patients were considered for inclusion with 168 subjects (44.4\%) ultimately included for analysis. The most common reason for exclusion was complete patient activity data not available during 
Table 1: Baseline demographics

\begin{tabular}{|c|c|c|c|}
\hline & All subjects, $\mathrm{n}=168$ & $\begin{array}{l}\text { Subjects with } \leq 8 \text { comorbidities and } \\
>2.2 \text { hours overall mean activity, } n=65\end{array}$ & $\begin{array}{l}\text { Subjects with }>8 \text { comorbidities and } \leq 2.2 \\
\text { hours overall mean activity, } n=27\end{array}$ \\
\hline Age, years (SD) & $63.0(22.8)$ & $65.0(10.3)$ & $74.7(11.8)$ \\
\hline Sex $\%$ male & $75.0 \%$ & $75.4 \%$ & $66.6 \%$ \\
\hline $\mathrm{BMI}, \mathrm{kg} / \mathrm{m}^{2}(\mathrm{SD})$ & $29.5(15.7)$ & $30.0(6.1)$ & $29.6(5.1)$ \\
\hline LVEF, \% (SD) & $34.5(28.8)$ & $37.9(12.6)$ & $36.9(13.3)$ \\
\hline Overall activity level, hours per day (SD) & $2.7(1.6)$ & $4.1(1.4)$ & $1.5(0.6)$ \\
\hline Aetiology, \% ischaemic & $57.7 \%$ & $44.6 \%$ & $70.4 \%$ \\
\hline Device type, \% ICD & $72.6 \%$ & $75.4 \%$ & $63.0 \%$ \\
\hline \multicolumn{4}{|l|}{ NYHA-FC } \\
\hline I & $48.8 \%$ & $61.5 \%$ & $29.6 \%$ \\
\hline III & $11.9 \%$ & $1.5 \%$ & $29.6 \%$ \\
\hline$\geq 1$ hospitalisations & $17.3 \%$ & $6.2 \%$ & $22.2 \%$ \\
\hline
\end{tabular}

$B M I=$ body mass index; ICD = implantable cardioverter defibrillator (versus bi-ventricular pacemaker); LVEF = left ventricular ejection fraction; NYHA-FC = New York Heart Association-Functional ClaSs; $S D=$ standard deviation.

sampling frame. Other reasons for exclusion were having a ventricular assist device, marked limitation in ambulatory ability, lower extremity joint replacement during the sampling frame, greater than five hospital admissions during the sampling frame, diagnosis of aggressive cancer during the sampling frame and documented prolonged time spent living out of state during the winter. Clinical characteristics of included patients can be found in Table 1.

\section{Inter-rater reliability and validity of patient activity measurement}

The intraclass correlation coefficient for the three investigators was high (0.996; 95\% confidence interval [Cl] 0.991, 0.998). ${ }^{1,2}$ Concurrent validity using the Pearson correlation coefficient was also excellent ( $r=0.995$, 0.998 and 0.995 for the three investigators, respectively).

\section{Seasonality of daily physical activity}

The seasonality of bi-monthly averages for daylight hours and average temperature are clearly evident in the autocorrelation plots (Figure $1 \mathrm{~A}$ and $B$ ). Similarly, bi-monthly averages of patient activity also clearly demonstrate seasonal variation (Figure $1 C$ ) that generally coincides with that of daylight hours and average temperature. However, the seasonal duration (i.e., time between peaks in the autocorrelation plots) for temperature and daylight was 6 months/12 lags, and the duration for patient activity was 4 months/8 lags. The association between seasonal change in daylight hours, average temperature, and patient activity is also evident in the graphs of the three variables over time (Figure 2). During the 2018 summer, average humidity and the resulting average heat index values were not substantially above $25^{\circ} \mathrm{C}$, and the timing of the warmest weeks did not appear to directly correlate with the start of the decrease in patient activity.

\section{Magnitude of seasonal differences in daily physical activity}

Based on Figure 2 and the mean bi-monthly values, the lowest patient activity occurred during the 2-week period around 1 February (2.6 hours per day/156 minutes per day) and the highest patient activity during the 2-week period around 1 June
(3.0 hours per day/180 minutes per day). The unadjusted/raw difference in average daily PA between 1 February and 1 June of 0.4 hours per day/24 minutes per day/2.8 hours per week/14.9\% was significantly different $(t=5.965, p<0.001)$. When excluding the 29 patients with hospitalisations during the sampling frame, the mean difference of 0.4 hours per day/24 minutes per day/2.8 hours per week $/ 15.4 \%$ was also statistically significant $(t=6.010, p<0.001)$.

\section{Presence of seasonal variation in patient activity controlling for clinical characteristics}

The Pearson correlation coefficient matrix revealed that only total number of comorbidities and overall mean activity level were significantly associated with the seasonal difference in patient activity and, therefore, were selected for inclusion in ANCOVA modelling. Repeated measures ANCOVA revealed a cubic effect of season on patient activity (within-subjects $F=9.806, p<0.000$ ) for the overall sample and when controlling for overall activity level $(F=14.65, \quad p<0.000)$. However, when controlling for the effect of comorbidities (dichotomised as $\leq 8$ as determined by the mean for the overall sample and an apparent breakpoint in the scatterplot for comorbidities and seasonal difference in patient activity) or number of comorbidities and overall activity level (dichotomised as $\leq 2.2$ hours per day), ${ }^{22}$ there was not a statistically significant effect of season on patient activity $(F=2.13, p=0.146$ and $F=0.762, p=0.638$, respectively). These confounding effects of overall activity level and comorbidities are illustrated in Figure 3 which demonstrates that those individuals with more comorbidities and lower overall activity levels did have a significant change in patient activity over the 1-year sampling period. Results are similar when excluding the 29 patients who had hospitalisations during the 1-year sampling period with one exception: there was not a statistically significant interaction effect of comorbidities and patient activity. Of note, there was a statistically significant correlation between total number of comorbidities and number of hospitalisations $(r=0.283, p<0.001)$. Additionally, those with $>8$ comorbidities demonstrated a statistically significant decline of 0.3 hours per day over the course of the year $(t=2.970, p=0.04)$ compared to the overall sample which demonstrated an overall decline of 0.14 hours per day $(t=2.474, p=0.014)$. 
Figure 1: Autocorrelation plots of average temperature $(A)$, daylight (B) and daily physical activity (C)

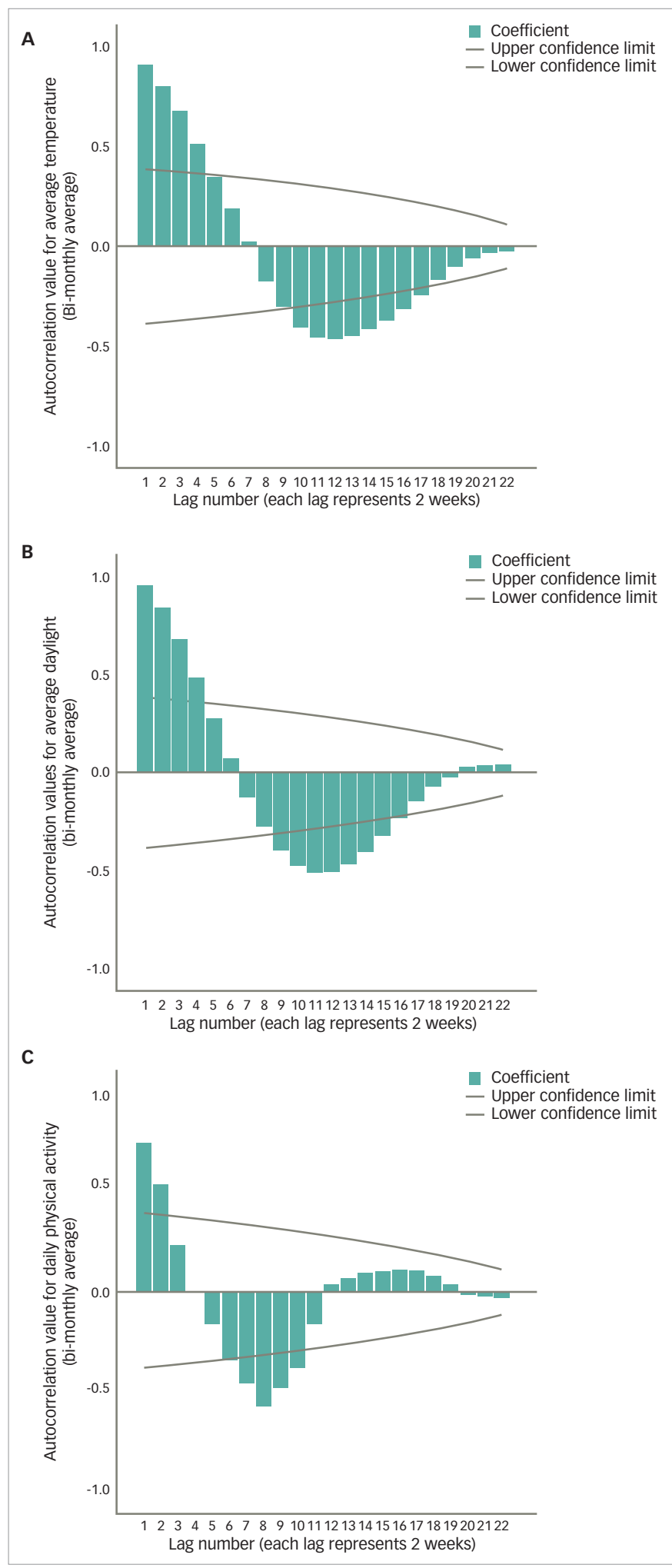

\section{Comparison of adjusted means for seasonal differences in patient activity}

Results of the one-way ANOVA to compare mean seasonal differences in patient activity adjusted for number of comorbidities and overall activity level are presented in Table 2. There was a main effect of the comorbidity-overall activity level grouping $(F=5.85, p=0.001)$ with Bonferroni post hoc tests revealing that the group with $\leq 8$ comorbidities and $>2.2$ hours per day of overall activity had greater seasonal variation
Figure 2: Plots of daily physical activity, temperature (A) and daylight (B)
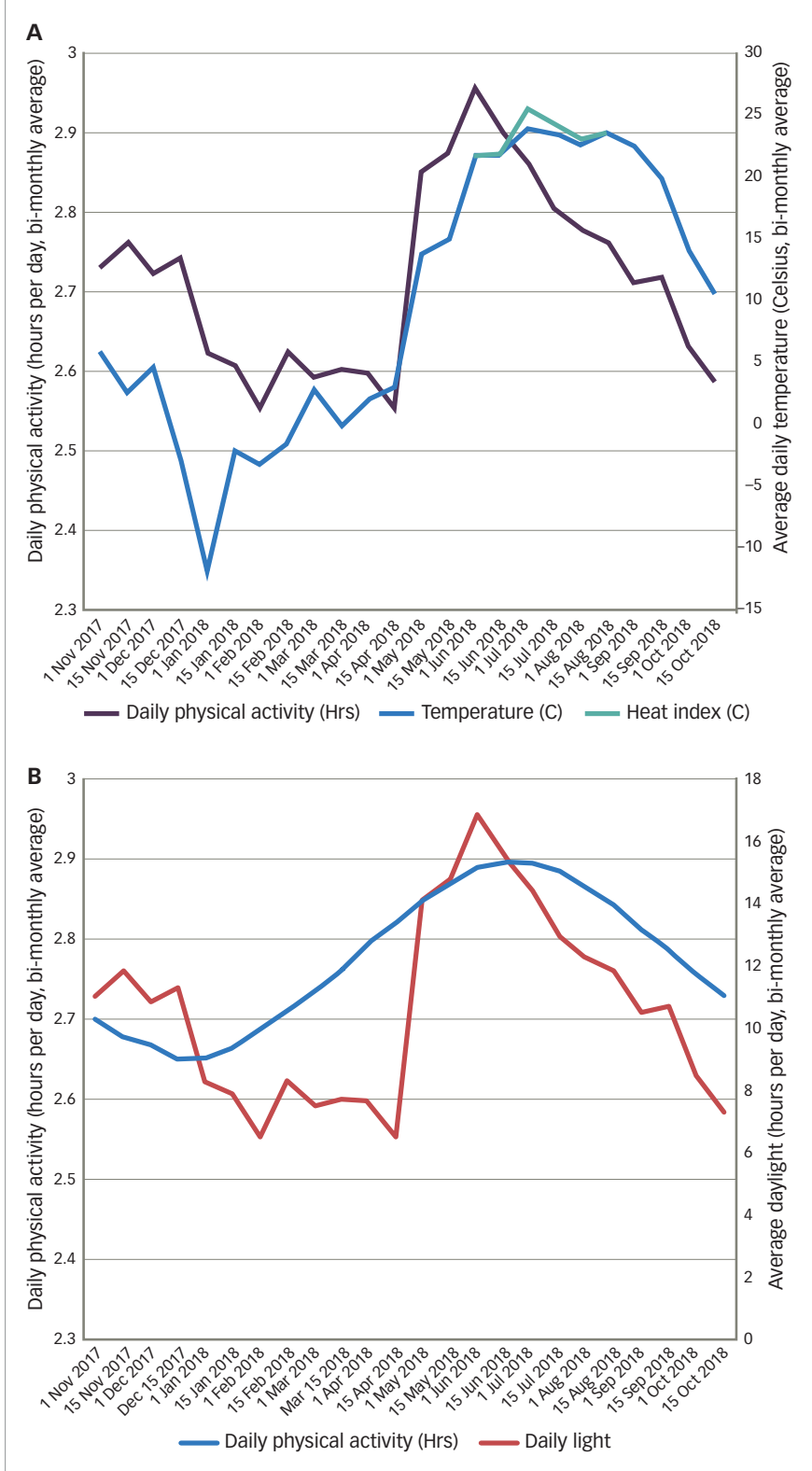

in patient activity than all other groups $(p<0.05)$, with a mean seasonal difference between February and June of 0.7 hour per day/42 minutes per day/4.9 hours per week.

\section{Discussion}

Previous research on seasonal variation in daily PA is limited by short daily PA sampling periods and use of non-longitudinal designs, and only one previous study included individuals with HF.19 Those studies that used short sampling periods are limited to conclusions about the short-term impact of weather parameters (temperature, daylight, precipitation). 57-10,12,16 Other studies made conclusions about seasonality based on non-longitudinal designs that compared separate groups of subjects from different seasons using relatively short sampling periods. ${ }^{1,2,1,1,1,15,17}$ This is problematic since the question of seasonality is most appropriately examined using time series data, ${ }^{24}$ which requires longitudinal sampling of the same individuals. Only seven previous studies have used this type of Iongitudinal design. 3,4,6,13,18,19,25,26 The present study used longitudinal data from a single cohort of patients with HF and implanted ICD/CRT devices. 
Estimated marginal means based on overall daily physical activity level (A) and total number of comorbidities (B)

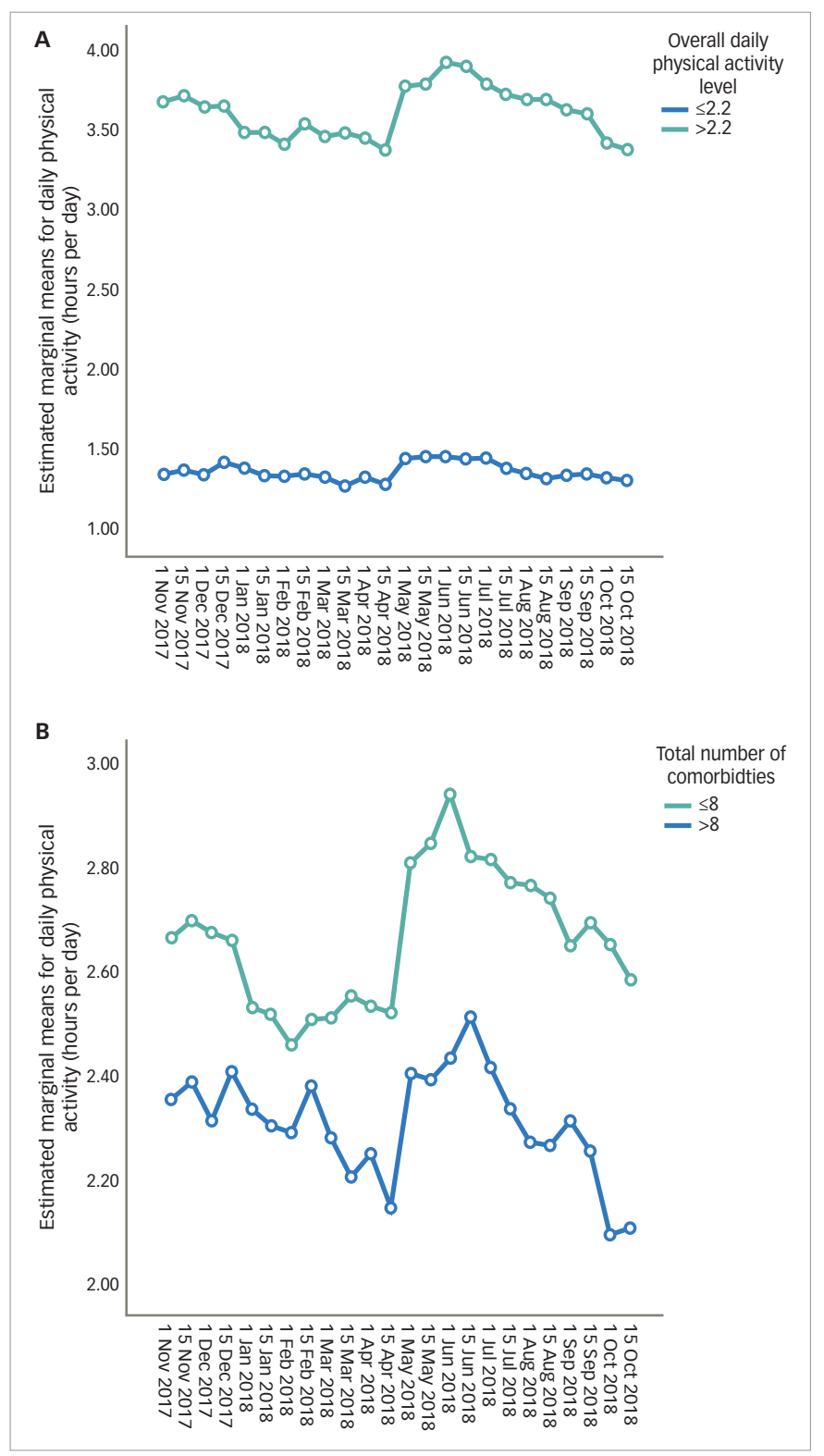

\section{Presence of seasonal variation in daily physical activity}

The seasonal effect on PA is consistent with Shoemaker et al. ${ }^{19}$ who observed similar trends in similar patients with HF and ICD/CRT devices using a small pilot sample. Sewell et al. ${ }^{2}$ and Furlanetto et al. ${ }^{5}$ both reported an effect of season on older subjects with COPD, as these studies both observed significantly decreased step counts during the winter months compared to summer months. Similarly, multiple other studies reported lower average daily PA during the winter months in older adults without $\mathrm{HF}^{-6,7,9,10,12,14,16,17}$ The authors of the present study are not aware of any previous studies that examined seasonal variation of daily PA in individuals with implanted devices but without HF, nor any studies of patients with HF but without implanted devices.

Potential variables that might account for the observed seasonal variation in daily PA are average temperature and hours of daylight. In the present study, the timing and duration of seasonal change for patient activity was slightly different than that of temperature and daylight hours; therefore, conclusions about the cause of seasonal variation in
Table 2: Mean seasonal difference in daily physical activity adjusted for total number of comorbidities and overall daily physical activity level

\begin{tabular}{|l|l|l|}
\hline Comorbidities and activity & $\mathrm{N}$ & Mean hours (SD) \\
\hline$>8$ comorbidities, $\leq 2.2 \mathrm{hrs} /$ day & 27 & $0.1(0.72)$ \\
\hline$>8$ comorbidities, $>2.2 \mathrm{hrs} /$ day & 28 & $0.2(0.77)$ \\
\hline$\leq 8$ comorbidities, $\leq 2.2 \mathrm{hrs} /$ day & 48 & $0.2(0.60)$ \\
\hline$\leq 8$ comorbidities, $>2.2 \mathrm{hrs} /$ day & 65 & $0.7(1.05)^{*}$ \\
\hline$* 0<0.05$ compared to all other groups & & \\
\hline
\end{tabular}

${ }^{*} p<0.05$ compared to all other groups.

hrs = hours; $S D=$ standard deviation.

daily PA cannot be made. In a previous study performed by Togo et al., ${ }^{6}$ daily PA was found to be more strongly associated with mean ambient temperature than day length. However, Klenk et al., ${ }^{11}$ Hagströmer et al., ${ }^{13}$ and Witham et al. ${ }^{7}$ found associations between daily PA and both temperature and daylight hours.

\section{Magnitude of seasonal variation in daily physical activity}

In the present study, the difference between the lowest daily PA in winter months and highest in summer months in the Midwestern USA was $14.9 \%$. This is consistent with previous pilot data from patients with $\mathrm{HF}$ and ICD/CRT devices in the Midwestern USA which ranged from 13.8-20.7\% (0.3-0.4 hours per day). ${ }^{19}$ In individuals without HF, previous studies have reported seasonal differences of $6.1-25.0 \%$ in Sweden, ${ }^{13}$ $7.1 \%$ in Norway, ${ }_{1}^{16}<10.0 \%$ in the south-eastern USA, ${ }^{25} 12.0 \%$ in Belgium, ${ }^{5}$ $15.0 \%$ in the United Kingdom, ${ }^{18} 15.0-20.0 \%$ in Michigan, ${ }^{15} 16.5 \%$ in Scotland, ${ }^{7}$ 20.0\% in Brazil, ${ }^{5} 23.5 \%$ in Japan, ${ }^{17}$ and $30.0 \%$ in Canada. ${ }^{14}$ This relatively large range of estimates for magnitude of seasonal variation in daily PA could be related to samples studied, geographic region and local climate, methods of daily PA measurement (objective versus subjective), daily PA measure used (step counts, energy expenditure, hours of activity, etc), or lack of control for confounding clinical characteristics.

\section{Seasonal variation in daily physical activity adjusted for clinical characteristics}

The repeated measures ANCOVA affirms the cubic (i.e., sinusoidal/not linear) main effect of time on daily PA which can be observed in Figure 2. However, that difference in daily activity between 1 February and 1 June is markedly obtunded in those with $>8$ comorbidities and $\leq 2.2$ hours per day of overall activity as is evident in Figure 3 . That is, when adjusting for comorbidities and overall PA levels, those with low comorbidities and higher activity levels are disproportionately affected by season with an average difference of 0.74 hours per day between the winter and summer months (February-June). Daily PA in all other patients varied by $<0.24$ hours per day. Aspvik et al. similarly observed a greater effect of temperature on daily PA in 'fitter' older adults. ${ }^{16}$ However, no prior study has adjusted for the confounding effect of comorbidities when examining seasonal variation in daily PA.

\section{Clinical and research relevance}

The results of the present study have several important clinical and research implications. First, maintaining daily PA in patients with HF and ICD/CRT devices, especially in those who are relatively more active and have fewer comorbidities, is a challenge in the winter months. Although this subgroup generally has a lower risk of frequent hospitalisations and lower mortality, there is an increase in hospitalisations and mortality during cold-weather months in patients with HF when compared to warmer months, ${ }^{27,28}$ and lower daily PA 
is associated with lower 11-month event-free survival. ${ }^{29}$ Further, maintaining daily PA over time warrants additional investigation given the observed decline over the course of the year, especially in those with $>8$ comorbidities.

A second clinical implication of the present study is that interpretation of the patient activity measure on the Cardiac compass report should consider season so as not to over-interpret improvements or declines in daily PA. Finally, daily PA prescription needs to consider an individual's unstructured and structured daily PA preferences and provide appropriate alternatives especially if those preferences include outdoor activities where hours of daylight and/or temperature may have an impact.

With regard to the research implications of the present study, studies investigating interventions to improve daily PA need to account for the effect of seasonality in the timing of patient enrolment and follow-up measurement. For example, sewell et al. were unable to account for the confounding effect of season on the percent change in patients with COPD following pulmonary rehabilitation. ${ }^{2}$ Similarly, Shoemaker et al. were unable to account for the confounding effect of seasonal variation on interventions to improve daily PA in patients with HF and ICD/CRTs. ${ }^{30}$ This is especially important when using rolling enrolment where changes in daily PA may be differentially impacted based on timing of enrolment, which could mask or accentuate intervention and control-group effects. Furthermore, accounting for the effect of season may also need to adjust for individual subject characteristics (i.e., comorbidities and overall activity level). Future research to develop and validate regression models to accurately adjust daily PA outcome measures based on season, meteorological variables, and clinical characteristics is needed.

Lastly, an important clinical and research implication of the present study is that of interpretation of clinically meaningful change. There are currently only a few estimates of clinically meaningful change in daily PA in individuals with HF. One minimum clinically important difference (MCID) estimate of daily PA in patients with HF and ICD/CRT devices was $20.0-41.5 \%\left(0.5-1.0\right.$ hours per day) ${ }^{23}$ Another study in patients with HF using external accelerometers, observed poor alignment of the MCID (a measure of change perceptible by a patient) of $14.6 \%$ (0.5-1.0 hours per day) and the minimum detectable difference (a measure related to measurement error) of $64 \% .{ }^{22}$ However, despite the lack of a clearly established threshold for clinically meaningful change in daily PA in patients with $\mathrm{HF}$, the amount of seasonal variation identified in the present study, especially in those who are more active and have fewer comorbidities, could significantly confound interpretation of change in an individual patient clinically and a group of subjects in a research study.

\section{Limitations}

The present study is not without limitations. The analyses in the present study used a visual estimation of bi-monthly daily activity from a graph as Medtronic proprietary software is required to extract the raw/daily data. Although visual estimation was demonstrated to be reliable and accurate in a small subgroup of our sample, replication of the present study using the raw/daily data points in another group of similar patients is necessary. The present study excluded a large number of patients based on criteria that would confound the effect of season on daily PA and we did not record the number of subjects in each exclusion criterion, though the majority were excluded due to incomplete data. Although the strict exclusion criteria were necessary to better isolate the effect of season by eliminating potential confounding variables, it also limits generalisability of the findings.

Devices from Medtronic are single-axis accelerometers that only record activity data in the sagittal plane. These devices require a threshold of approximately 70-80 steps/min to record activity; therefore, non-ambulatory activities would not be accounted for. Additionally, it is unclear whether restricting the sample to individuals with Medtronic devices limits generalisability to other similar individuals with devices from other manufacturers. However, including individuals with other devices that have different measurement mechanisms and associated algorithms could confound the data.

The observed downward trajectory of daily PA in those individuals with $>8$ comorbidities could confound the inferences made about seasonal differences. This could explain why there was not an effect of season when controlling for comorbidities. Unless noted in a patient's chart, we were unable to account for those that spend a portion of the year outside of the west Michigan area which might affect daily PA or whether a patient was hospitalised in another health system. Additionally, patients could have fallen ill at some point during the sampling period, but not ill enough to warrant hospitalisation. Lastly, the results of this study cannot be generalised to all patients with HF as it was specific to those with Medtronic devices and west Michigan weather and seasonality.

\section{Conclusions}

The present study affirms the seasonality of daily PA in a cohort of patients with HF and ICD/CRT devices, with an overall difference between the winter and summer months of 0.4 hours per day/24 minutes per day/2.8 hours per week/14.9\%. This seasonal effect on daily PA is significantly greater in those with $\leq 8$ comorbid conditions and overall activity level $>2.2$ hours per day. Seasonal variation should be accounted for when interpreting change in daily PA in clinical practice and when designing and interpreting results of clinical trials investigating interventions to improve daily PA. •
1. Izawa KP, Watanabe $\mathrm{S}$, Brusaker $\mathrm{PH}$, et al. Leisure time physical activity over four seasons in chronic heart failure patients. Int J Cardiol. 2014;177:651-3.

2. Sewell L, Singh SJ, Williams JE, Morgan MD. Seasonal variations affect physical activity and pulmonary rehabilitation outcomes. J Cardiopulm Rehabil Prev. 2010;30:329-33.

3. Alahmari AD, Mackay AJ, Patel ARC, et al. Influence of weather and atmospheric pollution on physical activity in patients with COPD. Respir Res. 2015;16:71-80

4. Pitta F, Breyer MK, Hernandes NA, et al. Comparison of daily physical activity between COPD patients from Central Europe and South America. Respir Med. 2009;103:421-6.

5. Furlanetto KC, Demeyer H, Sant'anna T, et al. Physical activity Furlanetto KC, Demeyer H, Sant'anna T, et al. Physical activity of patients with COPD from religions with different climatic variations. Int J Chron Obstruct Pulmon Dis. 2017;14:276-83.

Togo F, Watanabe E, Park H, et al. Meteorology and the physica activity of the elderly: the Nakanojo study. Int I Biometeorol. 2005:50:83-9.

7. Witham MD, Donnan PT, Vadiveloo T, et al. Association of day length and weather conditions with physical activity levels in older community dwelling people. PLoS One. 2014;9:e85331.

8. Jones GR, Brandon C, Gill DP. Physical activity levels of community-dwelling older adults are influenced by winter weather variables. Arch of Geron Geria. 2017;71:28-33.

9. Brandon CA, Gill DP, Speechley M, et al. Physical activity levels of older community dwelling adults are influenced by summe weather variables. Appl Physiol Nutr Metab. 2009;34:182-90

10. Hasegawa J, Suzuki H, Yamauchi T. Impact of season on the association between muscle strength/volume and physical activity among community-dwelling elderly people living in snowy-cold regions. J Physiol Anthropol. 2018;37:25.

11. Klenk J, Büchele G, Rapp K, et al. ActiFE Study Group. Walking on sunshine: effect of weather conditions on physical activity in older people. J Epidemiol Community Health. 2012;66:474-6.

12. Chan CB, Ryan DAJ, Tudor-Locke C. Relationship between objective measures of physical activity and weather: a objective measures of physical activity and weather. a

13. Hagströmer M, Rizzo NS, Sjöström M. Associations of season Hagstromer M, Rizzo NS, Sjostrom M. Associations of season
and region on objectively assessed physical activity and sedentary behaviour. J Sport Sci. 2014;32:629-34

14. Merchant AT, Dehghan M, Akhtar-Danesh N. Seasonal variation in leisure time physical activity among Canadians. Can J Public Health. 2007;98:203-8.

15. Pivarink JM, Reeves MJ, Rafferty AP. Seasonal variation in adult leisure-time physical activity. Med Sci Sports Exerc. 2003;35:1004-8

16. Aspvik NP, Viken $\mathrm{H}$, Ingebrigtsen JE, et al. Do weather changes influence physical activity level among older adults - the generation 100 study. PLOS One. 2018;13:e0199463.

17. Ogawa S, Seko T, Ito T, Mori M. Differences in physical activity between seasons with and without snowfall among elderly individuals residing in areas that receive snowfall. J Phys The Sci. 2019;31:12-6.

18. Sumukadas D, Witham M, Struthers A, McMurdo M. Day length and weather conditions profoundly affect physical activity levels in older functionally impaired people. J Epidemiol community Health. 2009;63:305-9.

19. Shoemaker M, Roper SE, Calkins TN. Seasonal variation of daily physical activity in individuals with heart failure. Heart Int. 2016;11:e25-31.

20. Kapteyn A, Banks J, Hamer M, et al. What they say and what they do: comparing physical activity across the USA, England, and the Netherlands. J Epidemiol Community Health 2018;0:1-6.

21. Shoemaker MJ, Cartwright $K$, Hanson $K$, et al. Concurrent validity of daily activity data from Medtronic ICD/CRT devices 
and the Actigraph GT3x triaxial accelerometer: a pilot study. Cardiopulm Phys Ther J. 2017;28:3-11. 22. Shoemaker MJ, Curtis A, Paul R, et al. Longitudinal daily activity patterns in individuals with chronic heart failure: derivation and association with prognosis and clinical characteristics. Cardiopulm Phys Ther J. 2013;24:25-33

23. Shoemaker MJ, Curtis AB, Vangsnes E, et al. Analysis of daily activity data from implanted cardiac defibrillators: the minimum clinically important difference and relationship to mortality/life expectancy. World I Cardiovasc Dis. 2012;2:129-35.

24. NIST/SEMATECH e-handbook of Statistical Methods. Available at: www.itl.nist.gov/div898/handbook/ pmc/section4/pmc443.htm (accessed 24 April 2018).

25. Tudor-Locke C, Bassett Jr DR, Swarts AM, et al. A preliminary study of one-year of pedometer self-monitoring. Ann Behav Med. 2004;28:158-62.

26. Bergstrahl EJ, Mehrsheed S, Offord KP, et al. Effect of season on physical activity score, back extensor muscle strength, and lumbar bone mineral density. J Bone Miner Res. 1990;5:371-7

27. Goggins W, Chan E. A study of the short-term association between hospital admissions and mortality from heart failure and meteorological variables in Hong Kong weather and heart failure in Hong Kong. Inter J Cardio. 2017;228:537-42.

28. Stewart S, McIntyre K, Capewell S, McMurray J. Heart failure in a cold climate: seasonal variation in heart failure-related morbidity and mortality. J Amer Coll Card. 2002;39:760-6.

29. Howell J, Strong BM, Weisenberg J, et al. Maximum daily 6 minutes of activity: an index of functional capacity derived from actigraphy and its application to older adults with heart failure. J Am Geriatr Soc. 2010;58:931-6.

30. Shoemaker MJ, Oberholtzer NL, Jongekrijg LE, et al. Exercise- and psychosocial-based interventions to improve daily activity in heart failure: a pilot study. Home Health Care Management and Practice. 2017;29:111-20. 